\title{
Invasive Cryptococcal Meningitis Presenting as a Skull Base Mass in An Immunocompetent Host: A Case Report
}

\author{
${ }^{1}$ Department of Skull Base and Minimally Invasive Neurosurgery, \\ Swedish Neuroscience Institute, Seattle, Washington, United States \\ 2 Department of Neuroanatomy, Seattle Science Foundation, Seattle, \\ Washington, United States \\ 3 Department of Neurological Surgery, Hospital Santo Tomas, \\ Panama, Panama \\ J Neurol Surg Rep 2019;80:e31-e35.
}

Joshua Prickett ${ }^{1}$ Juan Altafulla1,2,3 Anna Knisely ${ }^{1}$ Zachary Litvack ${ }^{1}$

\begin{abstract}
Keywords

- skull base

- cryptococcus

- meningitis

Introduction Encephalitis due to Cryptococcus neoformans has been seen almost exclusively in patients with severe compromise of their immune systems, such as acquired immune deficiency syndrome (AIDS). Fungal sinusitis with frank invasion through the cranial base and subsequent seeding of the central nervous system is rare, but should be considered in the differential of patients presenting with meningitis and sinus/skull base lesions even without obvious immune compromise. Improvements in diagnostic testing has increased the ability to correctly identify and new antimicrobials have allowed a condition that once carried a high morbidity and mortality to be managed with better outcomes. We present our treatment algorithm for successful management of an immunocompetent patient with extensive fungal encephalitis due to erosion through the skull base.

Case Description The patient is a 59-year-old male presenting unresponsive with sphenoid mass erosive of the skull base and symptoms of meningitis and encephalitis due to C. neoformans. Magnetic resonance imaging (MRI) at presentation demonstrated extensive diencephalic invasion, and a sphenoid mass with erosion of the skull base. Lumbar puncture (LP) confirmed elevated opening pressure of $45 \mathrm{~cm} \mathrm{H2O}$, and cultures confirmed infection with $C$. neoformans. He underwent operative sinonasal debridement followed by placement of an external ventricular drain for management of hydrocephalus. He was treated aggressively with a combination of both intravenous (IV) amphotericin B daily and intrathecal amphotericin B via the ventriculostomy thrice weekly. By the 2 nd week of treatment, patient regained consciousness. After 4 weeks of therapy, cerebrospinal fluid (CSF) cultures turned negative, and the external ventricular drain (EVD) was converted to a ventriculoperitoneal shunt (VPS) to manage chronic postinfectious hydrocephalus. We also placed a contralateral Ommaya reservoir to permit continued weekly intrathecal amphotericin B without violation of the shunt valve. With each instillation, the shunt was set to its highest setting to minimize CSF egress for 6 hours then reset to its "drainage" setting. After an additional 6 weeks of outpatient therapy, intrathecal therapy was discontinued. We continued CSF surveillance via Ommaya sampling monthly. At 9-month follow-up, he has remained clinically stable without evidence of recurrent infection. He has residual mild cognitive deficits, but is living semiindependently with his brother.
\end{abstract}

\author{
Address for correspondence Juan Altafulla, MD, Department of \\ Neuroanatomy, Seattle Science Foundation, 550 17th Avenue Suite \\ 600, Seattle, WA 98122, United States \\ (e-mail: juanaltafulla.md@gmail.com).
}

received

November 14, 2018

accepted

January 18, 2019
DOI https://doi.org/

10.1055/s-0039-1687886. ISSN 2193-6358. (c) 2019 Georg Thieme Verlag KG Stuttgart · New York
License terms

(c) (1) $\ominus$ (\$) 
Conclusions Fungal sinusitis is uncommon, especially in those without significantly compromised immune systems. Invasive fungal meningitis resulting in meningitis and encephalitis is even rarer. The condition carries high morbidity and mortality that can only be mitigated with a multidisciplinary effort by neurosurgery, otolaryngology, and infectious disease specialists. While there are no clear treatment guidelines, we present an approach that may permit longer term independent survival.

\section{Introduction}

Intracranial complications of peripheral infections are becoming more common and can be associated with infections of the frontal, ethmoid and sphenoid sinuses. Maxillary sinus infection is rarely seen extending into the central nervous system, although there has been reports of odontogenic maxillary sinus infections spreading into the central nervous system (CNS). ${ }^{1}$ Meningitis being the most common complication of all, symptomatology includes the triad of intense headache, fever, and focal neurological deficit. A study performed by Younis et al evaluated 82 patients that presented with sinusitis with complications in a 15 -year span, they noted that intracranial complications corresponded to 47\% (39 patients) of which 29 presented with meningitis. Cryptococcal meningitis was the common etiologic agent seen in AIDS (acquired immune deficiency syndrome) patients, whereas Streptococcus pneumoniae was the most common microorganism in patients without AIDS. ${ }^{2}$ Other complications include brain abscess, subdural empyema, cavernous sinus thrombosis, epidural abscess, osteomyelitis, and pituitary abscess has also been reported as an extremely rare finding. ${ }^{3}$ A multidisciplinary approach is preferred for treating these patients.

\section{Case Description}

Here, we describe a case of a 59-year-old male with a history of sarcoidosis. He presented to a referring emergency department with signs and symptoms of meningitis and altered mental status and was transferred to our institution. His chief complaint was headache with neck stiffness and photophobia as well as blurry vision. His sarcoidosis had been diagnosed by medistinoscopy 4 years prior after longstanding shortness of breath. Biopsy of enlarged hilar lymph nodes (-Fig. 1) was positive for noncaseating granulomas and he had remained symptomatically controlled with low dose oral prednisone. He had never smoked or used illicit drugs. His exam was normal except a lack of transillumination of the sinuses as well as mucosal erythema. Computed tomography (CT) was initially interpreted as a skull base mass suspicious for mucocele; however, closer inspection demonstrated a left sided dehiscence of the skull base. There was opacification of the middle and posterior left ethmoid air cells, posterior right ethmoid air cells, and bilateral sphenoid sinuses, as well as the left frontal sinus, as well as bone erosion involving the medial wall of the left orbit and the roof of the ethmoid air cells and planum sphenoidale (-Fig. 2). Magnetic resonance imaging (MRI) with and without contrast revealed cystlike expansion of numerous perivascular spaces throughout bilateral caudate nuclei and lentiform nuclei. A few enlarged cystic spaces are also present in bilateral thalami and midbrain, as well as diffuse leptomeningeal enhancement (-Fig. 3).

Given his presentation, he was started on empiric antibiotics including voriconazle and an lumbar puncture (LP) was performed which had an elevated opening pressure of $45 \mathrm{~cm}$ $\mathrm{H}_{2} \mathrm{O}$ and demonstrated monocytic and lympocytic pleocytosis with 130 white blood cells (WBC), slightly elevated protein at 47 with normal glucose of 42 . The initial differential included cryptococcus, histoplasma, cocidioides, blastomycosis, or candida. He was taken to the operating room (OR) on hospital day 2 for endoscopic endonasal exploration and debridement of fungal sinusitis with left maxillary antrostomy, total ethmoidectomy and sphenoidotomy with removal of right $(R)$ and left $(\mathrm{L})$ sphenoethmoidal contents. Intraoperatively, there was hemorrhagic fleshy white mass without obvious necrosis or "black turbinates." Frozen section demonstrated fungal organisms in mucosal tissue. The material was removed from the sinuses and there was no evidence of cerebrospinal fluid (CSF) leak upon Valsalva's maneuver.

Subsequent testing for HIV (human immunodeficiency virus) was negative. Infectious disease consultation was requested and he was started empiric liposomal amphotericin B with 5-flucytosine. Cryptococcal antigen titer from the CSF was positive at $>1: 2,560$, the upper limit of measurement. Culture from CSF and from intraoperative samples of sinus material isolated and confirmed Cryptococcus neoformans (-Fig. 4).

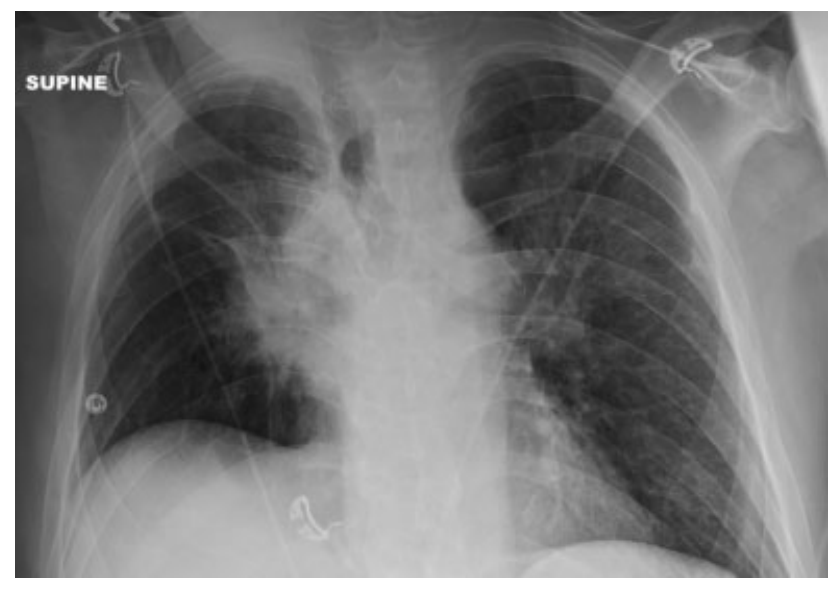

Fig. 1 X-ray image showing enlarged hilar lymph nodes. 




Fig. 2 Computed tomography showing opacification of the middle and posterior left ethmoid air cells, posterior right ethmoid air cells, and bilateral sphenoid sinuses, as well as the left frontal sinus, as well as bone erosion involving the medial wall of the left orbit and the roof of the ethmoid air cells and planum sphenoidale.



Fig. 3 Magnetic resonance imaging with and without contrast revealed cystlike expansion of numerous perivascular spaces throughout bilateral caudate nuclei and lentiform nuclei. A few enlarged cystic spaces are also present in bilateral thalami and midbrain consistent with cryptococcomas.
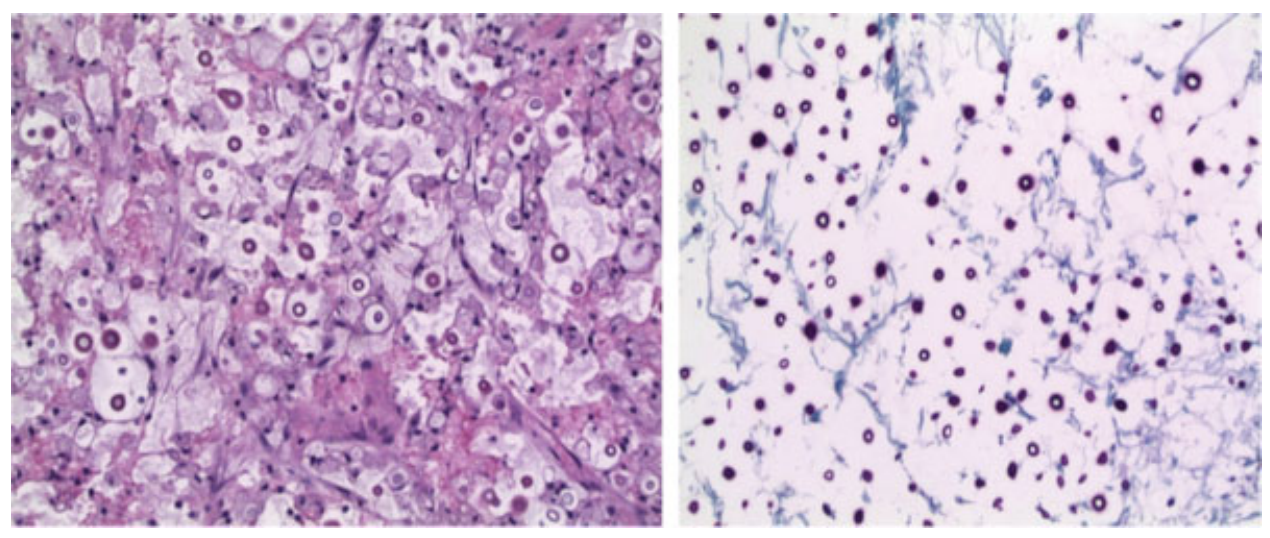

Fig. 4 The H\&E (haemtoxylin and eosin) on the left shows edema with a lack of an inflammatory response and numerous small round and refractile elements in the tissue concerning for infectious organisms. periodic acid schiff (PAS) stain highlights numerous organisms morphologically consistent with Cryptococcus neoformans.

Subsequent LPs demonstrated persistently elevated opening pressures, and the patient had decline in alertness necessitated placement of external ventricular drain on hospital day 7 with improvement in his neurologic exam. Given the severity of the CNS disease, he was started on intrathecal amphotericin thrice weekly via the external ventricular drain (EVD). Serial CSF analyses showed improvement in cryptococcal antigen titer and resolution of pleocytosis. Cultures remained positive postoperative days one and two, then remained sterile thereafter. He tolerated clamping of the EVD and it was removed; however, the developed symptoms of progressive decrease in alertness and lumbar 
puncture demonstrated elevated ICP thus a ventriculoperitoneal shunt (VPS) was placed with subsequent improvement. He was transferred to rehab facility for aggressive mobilization while continuing to receive a planned 6-week course of antifungals. The flucytosine had to be stopped due to thrombocytopenia, thus infectious disease felt it prudent to increase CNS concentration of amphotericin with intrathecal administration once again. To facilitate this without risking infection and loss of the VP shunt, an Ommaya reservoir was placed contralateral to the shunt. In a somewhat inventive fashion, after instillation of the amphotericin into the reservoir, the shunt valve was then set the highest setting to decrease drainage for several hours before being set back to allow enough drainage he did not develop symptoms of hydrocephalus.

\section{Discussion}

Acute invasive fungal sinusitis is most commonly seen in immunocompromised patients including solid organ transplant, uncontrolled diabetes, neutropenic patients from bone marrow suppression or aplasia, and advanced AIDS. Reports associating patients receiving small molecular kinase inhibitors, such as ibrutinib, for treating hematological malignancies that developed cryptococcal meningoencephalitis has recently been described in the literature. ${ }^{4,5}$ Other groups at risk for opportunistic infections are the organ transplant recipients with the incidence for cryptococcosis ranging from 0.3 to $5 \%{ }^{6}$ Regarding meningeal invasion, only a few cases describing cryptococcal sinusitis associated with organ recipient patients have been reported so far in the literature with only one linked to renal transplant. ${ }^{7}$

Clinical presentation of fungal sinusitis is variable; however, most commonly characterized by fever, facial pain, nasal congestion, and epistaxis. Fungal meningitis presents with fever, rigor, neck pain, headache, photophobia, followed by progressive obnulation and coma.

Most common pathogens include Aspergillus in neutropenic patients and the Zygomycetes, such as Mucor, in diabetic patients. The most common pathogen isolated in CSF of immunocompetent patients that presented with sinusitis are Streptococcus pneumoniae, followed by Staphylococcus aureus, Citrobacter sp., and Neisseria meningitides. Risk factors are associated with the development of intracranial complications besides sinus infection included frontal sinus fracture and periorbital cellulitis. ${ }^{2}$

Radiologic findings include sinus opacification with hypoattenuating mucosal thickening and does not have the hyperdense heterogeneity typical of noninvasive fungal mycetoma or allergic fungal sinusitis. Bony destruction and fat stranding may be present. Attention must be paid to orbital and intracranial invasion. In a review, pathologically confirmed IFS CT findings with patients with acute myelocytic leukemia and CT evidence of sinusitis, no reliable diagnostic radiologic finding was specific to IFS, although the authors conclude that severe unilateral thickening of the nasal cavity mucosa was the most consistent finding. ${ }^{8}$ Although bony erosion was not seen in the majority of this case series, bone erosion, extrasinus involvement, and intracranial spread are still cited as suggestive imaging findings. ${ }^{9,10}$

Middlebrooks et al presented an effective diagnostic imaging model for acute invasive rhinosinusitis, in which by using a seven-variable model to assess the lesion (periantral fat, bone dehiscence, orbital invasion, septal ulceration, pterygopalatine fossa, nasolacrimal duct, and lacrimal sac), they established a $100 \%$ specificity and $100 \%$ positive predictive value for invasive rhinosinusitis when two or more of this variables were present. ${ }^{11}$ Regarding imaging modalities, Groppo et al concluded that both MRI and CT have similar specificities (83 and $81 \%$, respectively) but sensitivity favored MRI with $86 \%$ compared with $69 \%$ obtained with $\mathrm{CT}$. $^{12}$

The mainstay of treatment of invasive fungal sinusitis is urgent aggressive medical and surgical management with correction of underlying immunocompromise. A multidisciplinary approach for treating these patients is fundamental in achieving a satisfactory outcome. CSF analysis and cultures are the pilar for diagnosis and focusing treatment to a specific pathogen. Initial management with empirical broad-spectrum antibiotics is used prior culture results; piperacillin/tazobactam and meropenem are the most commonly preferred, other combinations include vancomycin, mtronidazole, and cephotaxime. Once the culture results are available specific antibiotics are selected and target dose are optimized for penetrating the blood-brain barrier. ${ }^{13,14}$

Surgical options include endonasal approach for surgical debridment in the early stages of the disease, whereas open surgery is reserved for intraorbital extension, palatinal, and/ or intracerebral involvement. ${ }^{15}$ Mortality remains high, 50 to $80 \%$, especially in patients with complications.

\section{Conclusions}

Fungal sinusitis is uncommon, especially in those without significantly compromised immune systems. Invasive fungal meningitis resulting in meningitis and encephalitis is even more rare. The condition carries high morbidity and mortality that can only be mitigated with a multidisciplinary effort by neurosurgery, otolaryngology, and infectious disease specialists. While there are no clear treatment guidelines, we present an approach that may permit longer term independent survival.

\section{Funding Sources}

This research did not receive any specific grant from funding agencies in the public, commercial, or not-forprofit sectors.

Conflicts of Interest

None to disclose.

\section{References}

1 Courville CB, Rosenvold LK. Intracranial complications of infections of nasal cavities and accessory sinuses: a survey of lesions observed in a series of fifteen thousand autopsies. Arch Otolaryngol 1938;27(06):692-731 
2 Younis RT, Anand VK, Childress C. Sinusitis complicated by meningitis: current management. Laryngoscope 2001;111(08):1338-1342

3 Anagnos VJ, Hanel RA, Naseri I. Pituitary abscess: a rare clinical entity in the presence of acute rhinosinusitis: a case report. J Neurol Surg Rep 2018;79(02):e36-e40

4 Stankowicz M, Banaszynski M, Crawford R. Cryptococcal infections in two patients receiving ibrutinib therapy for chronic lymphocytic leukemia. J Oncol Pharm Pract 2018 (e-pub ahead of print)doi: $10.1177 / 1078155217752078$

5 Sun K, Kasparian S, Iyer S, Pingali SR. Cryptococcal meningoencephalitis in patients with mantle cell lymphoma on ibrutinib. Ecancermedicalscience 2018;12:836

6 Vilchez RA, Fung J, Kusne S. Cryptococcosis in organ transplant recipients: an overview. Am J Transplant 2002;2(07):575-580

7 Iyer SP, Movva K, Wiebel M, et al. Cryptococcal meningitis presenting as sinusitis in a renal transplant recipient. Transpl Infect Dis 2013;15(05):E187-E190

8 DelGaudio JM, Swain RE Jr., Kingdom TT, Muller S, Hudgins PA. Computed tomographic findings in patients with invasive fungal sinusitis. Arch Otolaryngol Head Neck Surg 2003;129(02):236-240

9 Toyama C. Invasive fungal sinusitis. In: Nunes RH, Abello AL, Castillo M, eds. Critical Findings in Neuroradiology. Switzerland: Springer International Publishing; 2016:285-291
10 Kinsella JB, Rassekh CH, Bradfield JL, et al. Allergic fungal sinusitis with cranial base erosion. Head Neck 1996;18(03):211-217

11 Middlebrooks EH, Frost CJ, De Jesus RO, Massini TC, Schmalfuss IM, Mancuso AA. Acute invasive fungal rhinosinusitis: a comprehensive update of $\mathrm{CT}$ findings and design of an effective diagnostic imaging model. AJNR Am J Neuroradiol 2015;36(08): 1529-1535

12 Groppo ER, El-Sayed IH, Aiken AH, Glastonbury CM. Computed tomography and magnetic resonance imaging characteristics of acute invasive fungal sinusitis. Arch Otolaryngol Head Neck Surg 2011;137(10):1005-1010

13 Szyfter W, Bartochowska A, Borucki Ł, Maciejewski A, KrukZagajewska A. Simultaneous treatment of intracranial complications of paranasal sinusitis. Eur Arch Otorhinolaryngol 2018;275 (05):1165-1173

14 Sonneville R, Ruimy R, Benzonana N, et al; ESCMID Study Group for Infectious Diseases of the Brain (ESGIB). An update on bacterial brain abscess in immunocompetent patients. Clin Microbiol Infect 2017;23(09):614-620

15 Kasapoglu F, Coskun H, Ozmen OA, Akalin H, Ener B. Acute invasive fungal rhinosinusitis: evaluation of 26 patients treated with endonasal or open surgical procedures. Otolaryngol Head Neck Surg 2010;143(05):614-620 